\title{
Antiangiogenesis Combined with Immunotherapy to Treat Advanced Small-Cell Carcinoma of the Esophagus Resistant to Chemotherapy: According to the Guidance of Next-Generation Sequencing
}

This article was published in the following Dove Press journal:

OncoTargets and Therapy

Man Jiang

Xiaochun Zhang

Cancer Precision Medical Center, The Affiliated Hospital of Qingdao University, Qingdao University, Qingdao, 266003,

People's Republic of China
Correspondence: Xiaochun Zhang Cancer Precision Medical Center, The Affiliated Hospital of Qingdao University, Qingdao University, 59 Haier Road, Qingdao, 266003, People's Republic of China

Email zhangxiaochun9670@I26.com

\begin{abstract}
A 64-year-old woman admitted to our hospital with the chief complaint of swallowing obstruction was diagnosed as relapsed small-cell carcinoma of the esophagus. Complete remission (CR) was observed after six cycles of irinotecan plus cisplatin therapy. According to the results of a next-generation sequencing analysis of the tumor specimen, anlotinib (12 mg PO q3w) was recommended. After 1 month of anlotinib treatment, the tumor decreased significantly according to computed tomography scan and gastroscopy. However, the disease progressed after 2 months of therapy. A gene analysis of the new puncture sample showed microsatellite instability and a high tumor mutation burden. Immunohistochemistry indicated positive programmed death ligand-1 expression $(>1 \%)$. Because of these results, the patient was treated with anlotinib (12 mg PO q3w) in combination with toripalimab (240 mg IV drip q3w). After 3 months of therapy, CR was achieved, although progression-free survival had not been reached at the time of publication.
\end{abstract}

Keywords: anlotinib, toripalimab, NGS, cell esophagus carcinoma

\section{Introduction}

Small-cell lung carcinoma (SCLC) has historically one of the highest malignancy rates of all tumor types. ${ }^{1}$ Anlotinib combined with PD-1 therapy has been approved in Chinese Clinical Guidelines for SCLC. By comparison, small-cell carcinoma of the esophagus (SCCE), which accounts for only $0.5 \%-2.8 \%$ of all esophageal malignancies,is a particularly rare tumor characterized by high malignancy, early metastasis, and poor prognosis, ${ }^{2}$ without classical therapy guidelines. Because of the low incidence of SCCE, the results of large-scale randomized controlled studies are obscure. ${ }^{3-10}$ Therefore, the optimal, standard treatment for limited-stage SCCE has not been established. Herein, we report a case of chemotherapy resistance in a patient who showed achieved complete remission (CR) to therapy with anlotinib, which is a tyrosine kinase-targeted agent, combined with toripalimab, which is an immune checkpoint inhibitor (ICI). This case mirrored the same pathological type in different cancers that were treated with the same therapy and achieved a good prognosis, ${ }^{11}$ thus providing a practical basis for "treating different diseases with the same therapy." 


\section{Method}

\section{Patient}

A 64-year-old woman was diagnosed with locally advanced SCCE 4 years prior. After four cycles of combination chemotherapy of irinotecan (100 mg, days 1 and 8) and cisplatin (50 mg, days 1 and 8), CR was achieved. After 2.5 years, the patient returned to our hospital for the sensation of swallowing obstruction. The patient was diabetic, but there was no history of hypertension or cardiovascular disease. Furthermore, no swelling of the superficial lymph nodes was detected by physical examination.

\section{Next-Generation Sequencing-Based Assay}

Tumor DNA was extracted from formalin-fixed paraffinembedded (FFPE) samples using QIAamp DNA FFPE Tissue Kit and Qiagen's DNEasy Blood and Tissue
Extraction Kit (Qiagen, Germany), respectively, according to the manufacturer's instructions. Genomic DNA from peripheral blood was purified using the QIAamp DNA Blood Mini Kit (Qiagen). All FFPE tissue samples were reviewed by a qualified pathologist to ensure $>70 \%$ tumor content. We performed next-generation sequencing (NGS) using OncoScreen Plus Kit (Burning Rock Biotech, Guangzhou, China), which is a panel consisting of 520 cancer-related genes spanning 1.64 Mb of the human genome in the NextSeq platform (Illumina, San Diego, CA, USA) (Table 1).

\section{Functional and Pathway Enrichment Analysis}

The Gene Ontology (GO) database (http://www.geneontol ogy.org) includes three main categories: biological process, cellular component, and molecular function. The Kyoto Encyclopedia of Genes and Genomes database

Table I NGS Results of Gastroscopy Puncture Samples Before and After Anlotinib Therapy

\begin{tabular}{|c|c|c|c|c|c|c|}
\hline \multirow[t]{2}{*}{ Gene } & \multicolumn{3}{|c|}{ Before Alotinib Therapy } & \multicolumn{3}{|c|}{ After Alotinib Therapy } \\
\hline & Base & Amino Acid & Frequency & Base & Amino Acid & Frequency \\
\hline BRCA2 & $c .8695 \mathrm{C}>\mathrm{T}$ & P.[Q2899*] & $2.01 \%$ & - & - & - \\
\hline RBI & c. $1510 C>T$ & p.Gln504* & $63.83 \%$ & c. $1510 \mathrm{C}>\mathrm{T}$ & p.Gln504* & $80.42 \%$ \\
\hline TP53 & $\begin{array}{l}\text { c. } 1024 \mathrm{C}>\mathrm{T} \\
\mathrm{c} .833 \mathrm{C}>\mathrm{A}\end{array}$ & $\begin{array}{l}\text { p.Arg342* } \\
\text { p.Pro278His }\end{array}$ & $\begin{array}{l}35.19 \% \\
36.09 \%\end{array}$ & $\begin{array}{l}\text { c. } 1024 \mathrm{C}>\mathrm{T} \\
\text { c. } 833 \mathrm{C}>\mathrm{A}\end{array}$ & $\begin{array}{l}\text { p.Arg342* } \\
\text { p.Pro278His }\end{array}$ & $\begin{array}{l}48.93 \% \\
42.67 \%\end{array}$ \\
\hline ATRX & c.2658_2659dup & p.Thr887fs & $25.34 \%$ & c.2658_2659dup & p.Thr887fs & $25.89 \%$ \\
\hline KMT2A & $c .5419 \mathrm{C}>\mathrm{G}$ & P.[Q।807E] & $39.36 \%$ & - & - & - \\
\hline PIK3CA & - & - & - & Amplification & $3 q 26.32$ & $\mathrm{CN}=4.45$ \\
\hline PIK3CB & c. $1275 \mathrm{~T}>\mathrm{A}$ & P. $[Y 425 *]$ & $21.84 \%$ & - & - & - \\
\hline EP300 & c. $4228 \mathrm{~A}>\mathrm{T}$ & p.Arg|4|0Trp & $33.15 \%$ & $\begin{array}{l}\text { c. } 4228 \mathrm{~A}>\mathrm{T} \\
\text { Amplification }\end{array}$ & $\begin{array}{l}\text { p.Arg|4|0Trp } \\
22 q 13.2\end{array}$ & $\begin{array}{l}81.48 \% \\
C N=6.78\end{array}$ \\
\hline TNFRSF8 & c. $443 \mathrm{~T}>\mathrm{C}$ & $\mathrm{p} \cdot[\mathrm{VI} 48 \mathrm{~A}]$ & $17.28 \%$ & - & - & - \\
\hline TNFAIP3 & - & - & - & c. I323del & p.Ala442fs & $34.34 \%$ \\
\hline CTNNBI & c.78G $>\mathrm{T}$ & p.Gln26His & $60.69 \%$ & - & - & - \\
\hline CSFIR & c. $1151 G>A$ & p.Arg384Lys & $42.21 \%$ & c. $115 \mid G>A$ & p.Arg384Lys & $60.69 \%$ \\
\hline FATI & - & - & - & $c .55 \mid 5 G>T$ & p.Glul839* & $90.49 \%$ \\
\hline PTK2 & - & - & - & Amplification & $8 q 24.3$ & $\mathrm{CN}=6.02$ \\
\hline sox2 & - & - & - & Amplification & $3 q 26.33$ & $\mathrm{CN}=5.87$ \\
\hline MYC & - & - & - & Amplification & $8 q 24.21$ & $\mathrm{CN}=5.26$ \\
\hline
\end{tabular}

Abbreviations: NGS, next-generation sequencing (OncoScreen Pluspanel, Nextseq); RBI, RB transcriptional corepressor I; CTNNBI, catenin beta-I; PI3K, phosphatidylinositol 3-kinases; TNFAIP3, tumor necrosis factor, alpha-induced protein 3; MYC, myelocytomatosis oncogene. 
(KEGG; http://www.genome.ad.jp/kegg) collects genomic, chemical, and systematic functional information. The Cluster Profiler package implements methods for analyzing and visualizing the functional profiles of genes and gene clusters. In this study, GO terms and KEGG pathways were analyzed using the Cluster Profiler package with an enrichment threshold of $P<0.05$.

\section{Results}

Histopathologic examination of gastroscopy biopsy specimens indicated relapsed SCCE (Figures 1A and 3A). Immunohistochemistry results showed positive staining of high molecular weight cytokeratin 56 (Figure 1B), alpha-synuclein (Figure 1C), and Ki-67 (80\%) (Figure 1D). We performed NGS testing but did not find any meaningful mutations (Table 1). According to these results, irinotecan (100 $\mathrm{mg}$, days 1 and 8$)$ and cisplatin (50 mg, days 1 and 8) chemotherapy was administered for another six cycles. However, computed tomography (CT) scan and gastroscopy indicated disease progression (Figure 2A) after intraperitoneal(IP) chemotherapy. The following NGS examination of the gastroscopy biopsy specimens indicated a significant increase in the mutation rates of BRCA2, RB1, TP53, ATRX, KMT2A, PIK3CB, EP300, TNFRSF8, CTNNB1, and CSF1R. Anlotinib, which is an oral tyrosine kinase inhibitor targeting the vascular endothelial growth factor receptor, has been approved as a third-line recommended therapy in the 2020 Chinese Society of Clinical Oncology (CSCO) guideline for SCLC. Considering the similar histopathology of the patient,12,13 anlotinib was suggested to the patient (12 mg PO $\mathrm{q} 3 \mathrm{w})$. After 1 month, both the CT scan and gastroscopy therapy showed that the esophageal wall had returned to normal (Figures 2B and 3B), with limited side effects (normal levels of transaminase, hypothyroidism, and grade I hyperlipidemia). After 3 months, the esophageal wall thickened again (Figures 2C and 3C). The third NGS assay showed significant mutation changes: The mutations BRCA2, KMT2A, PIK3CB, TNFRSF8, and CTNNB1 disappeared, whereas the mutations TNFAIP3 and FAT1 appeared. The mutation rates of RB1, TP53,
A
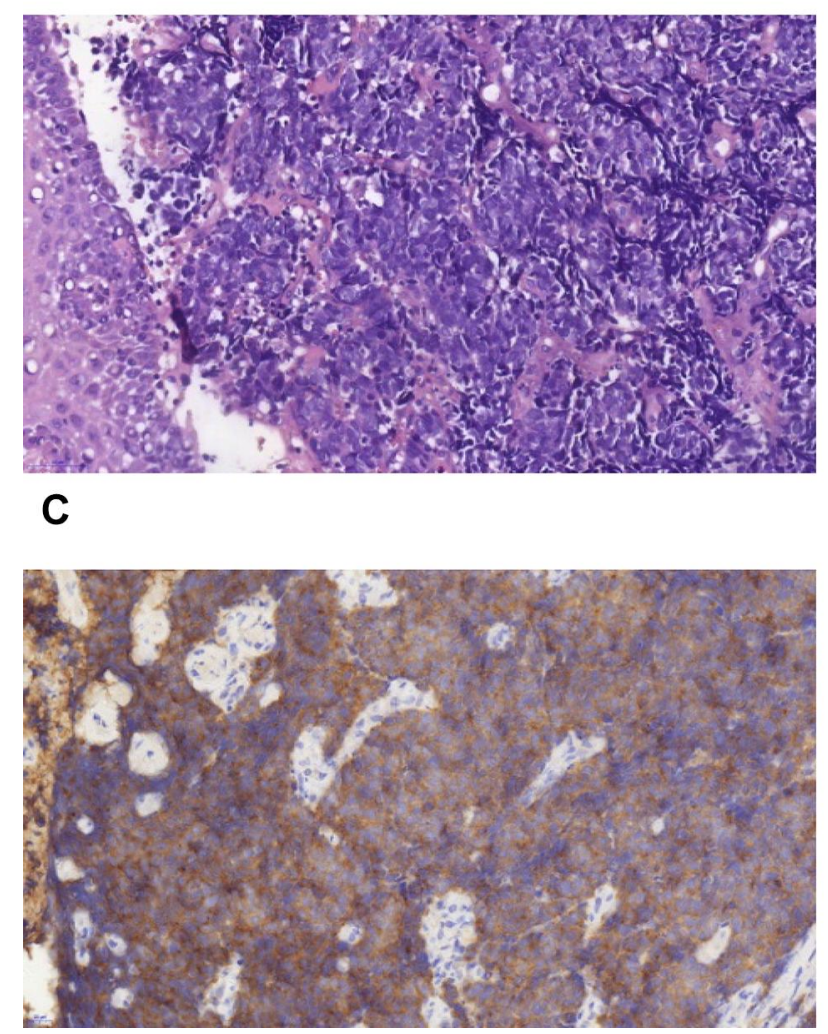

B
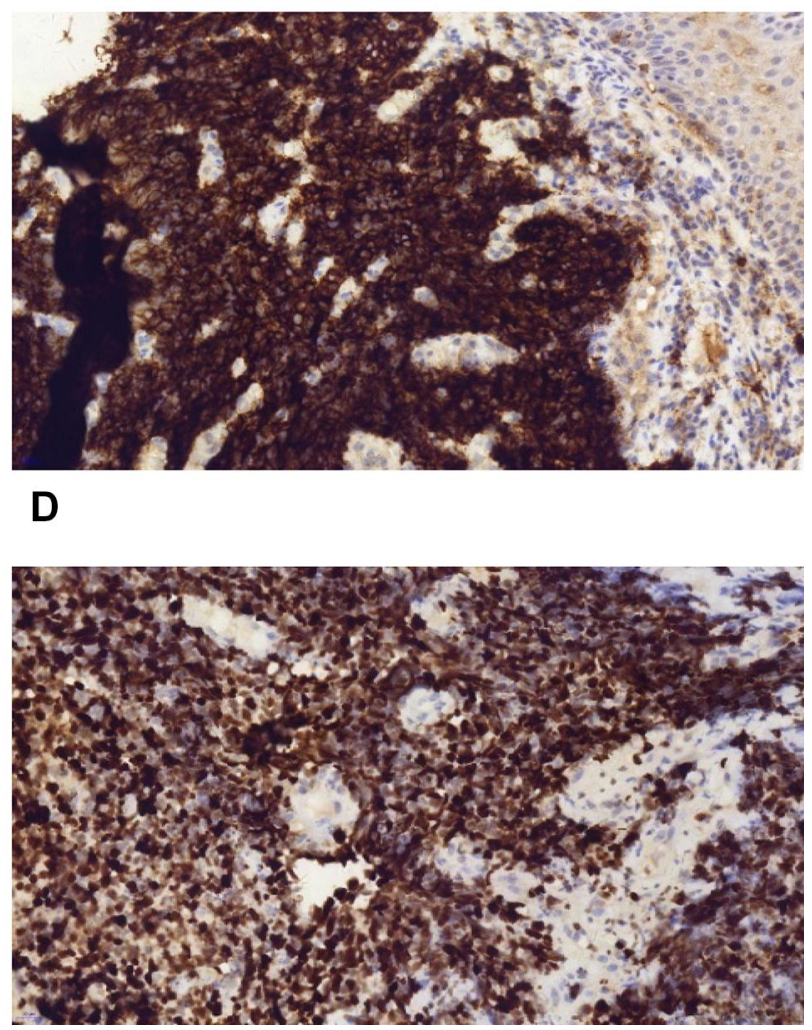

Figure I (A) Hematoxylin and eosin staining of biopsy specimens indicated SCCE (200x). (B) IHC showed positive staining of high molecular weight cytokeratin 56 with diffusion (200x). (C) IHC showed positive staining of alpha-synuclein (200x) Syn (+), and (D) Ki-67 (200x) 80\% positive. 
A

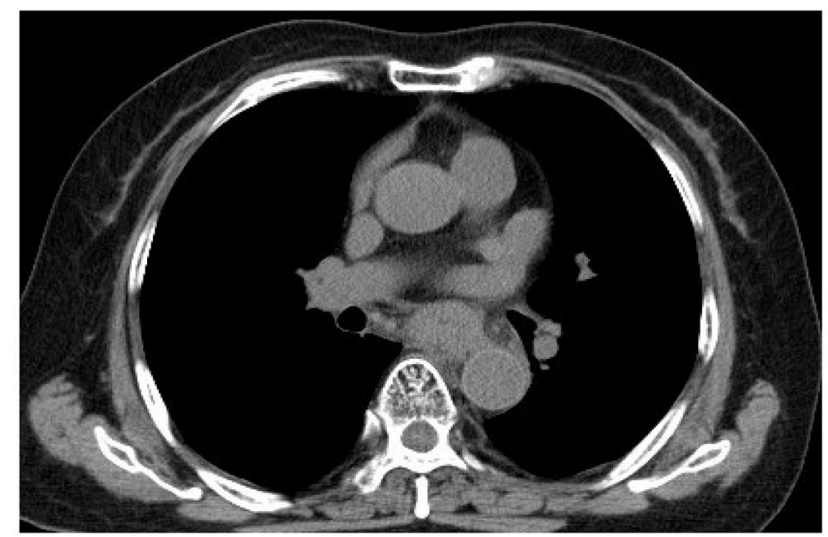

C

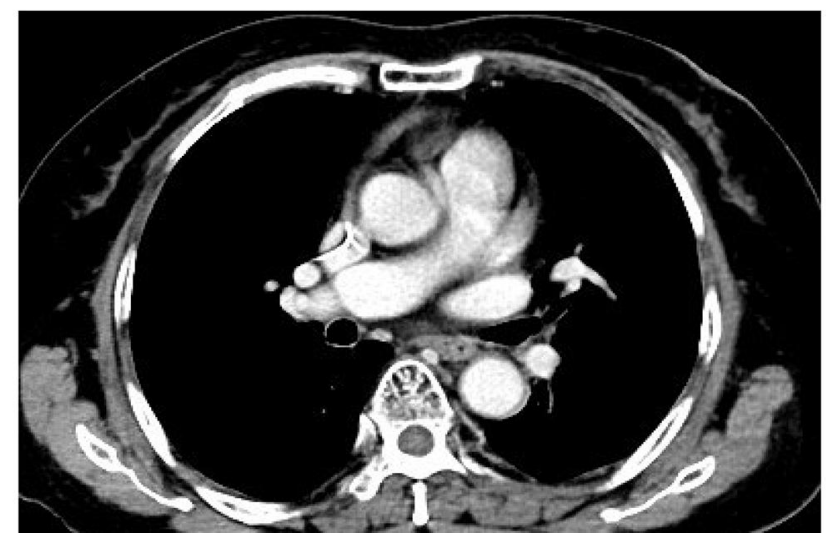

B

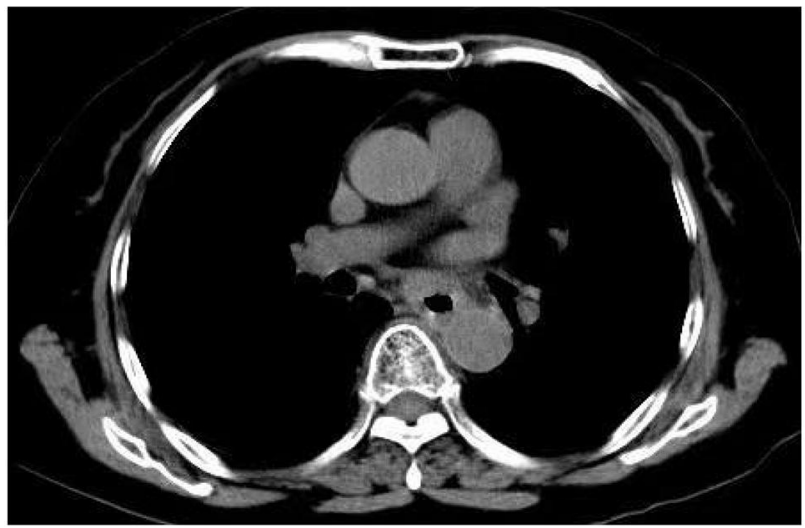

D

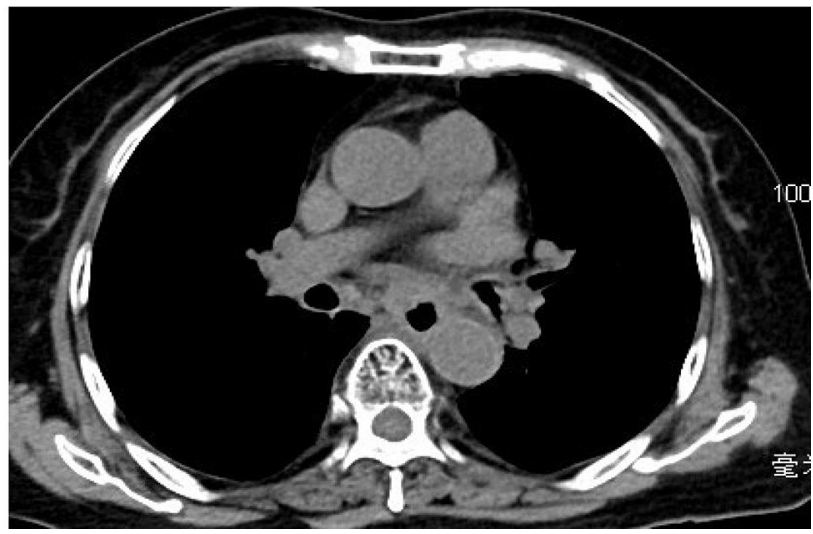

Figure 2 Chest CT scans during therapy. (A) SCCE relapsed after six courses of IP chemotherapy. (B) Esophageal wall shrinkage after I month of anlotinib monotherapy. (C) Esophageal wall re-thickening after 3 months of anlotinib monotherapy. (D) After 3 months of treatment with anlotinib in combination with toripalimab.

EP300, and CTNNB1 significantly increased, whereas those of CSF1R decreased. A copy number amplification was observed in PIK3CA, PTK2, SOX2, and MYC. A high tumor mutation burden (TMB) (57.1 mutations/ $\mathrm{Mb}$ ) was observed, and immunohistochemistry detected a positive programmed death ligand-1 expression rate (PD-L1 > 1\%) (Figure 4). Based on these results, this patient was an ideal candidate for treatment with ICIs. $^{14,15}$ For economic reasons, toripalimab (240 mg IV drips $\mathrm{q} 3 \mathrm{~W}$ ) was chosen as the ICI. Improvement of her physical condition was observed after one cycle of combined treatment, and the sensation of swallowing obstruction disappeared. After 3 months of therapy, the clinical response was characterized as CR (Figures 2D and $3 \mathrm{D})$. Until the time of publication, the patient has remained in $\mathrm{CR}$, and the progression-free survival (PFS) has not yet been reached after a follow-up at13 months (PFS $>13$ months). The patient signed informed consent forms, which allowed for the publication of the relevant clinical and imaging data from her case.

The GO and KEGG enrichment analyses of differentially expressed genes (DEGs) before and after anlotinib therapy were conducted using the Cluster Profiler package to further understand the function and mechanism of the therapy (Figure 5). In the GO analysis, DEGs mainly indicate leukocyte migration, extracellular matrix organization, extracellular structure organization, and cell-substrate adhesion $(10 / 10, P<0.005)$. According to the KEGG analysis, according to the GO analysis, the significant enrichment before therapy with anlotinib combined with toripalimab was the focus of focal adhesion kinase (FAK) $(7 / 10, P<0.005)$. 
A

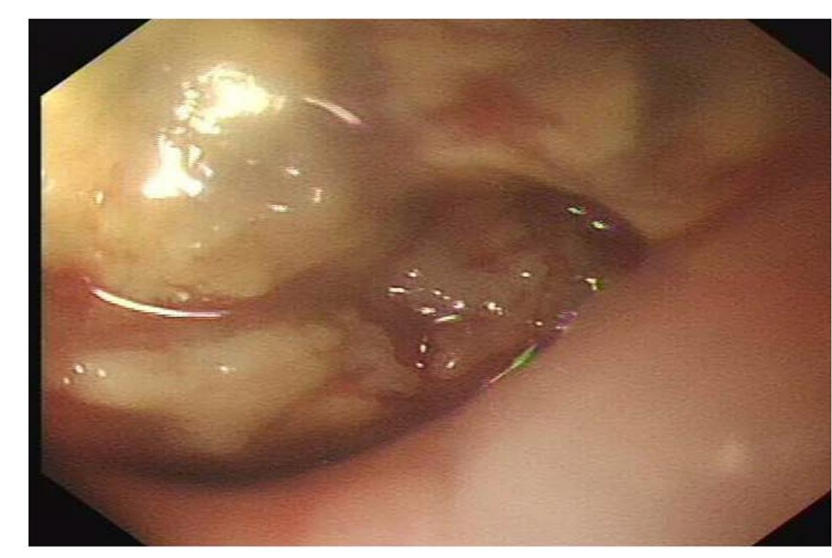

C

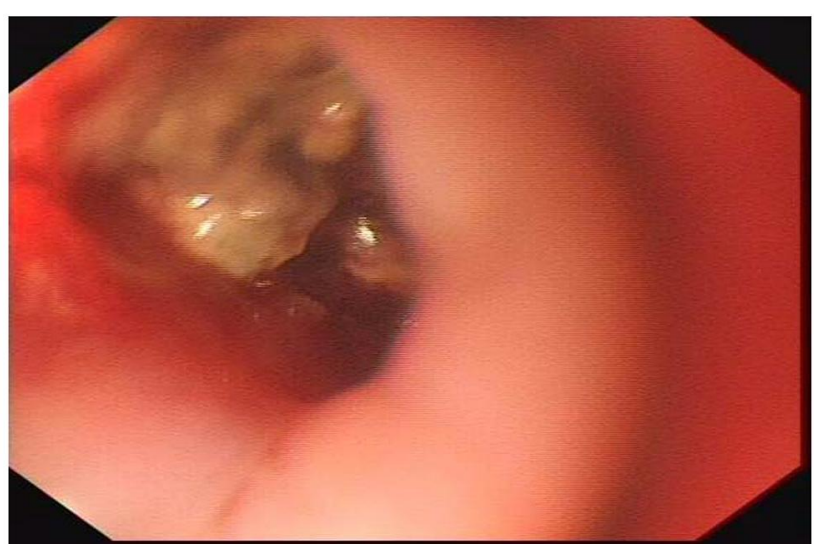

B

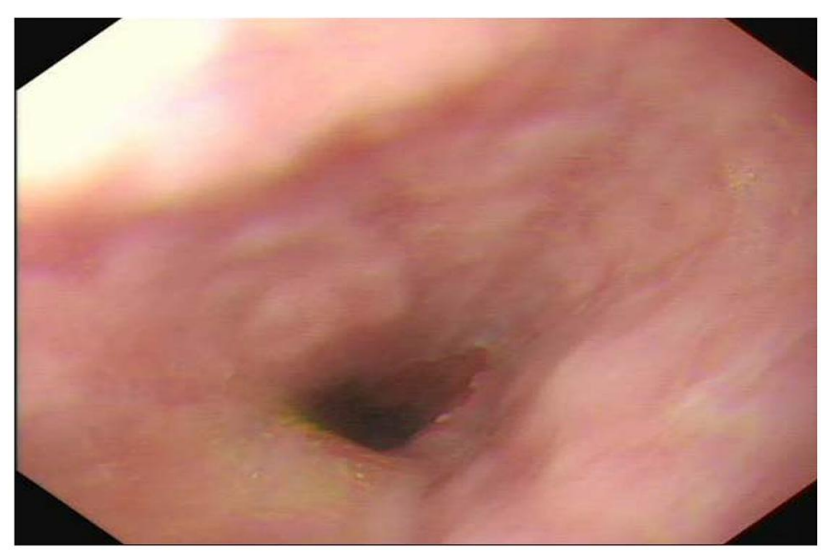

D

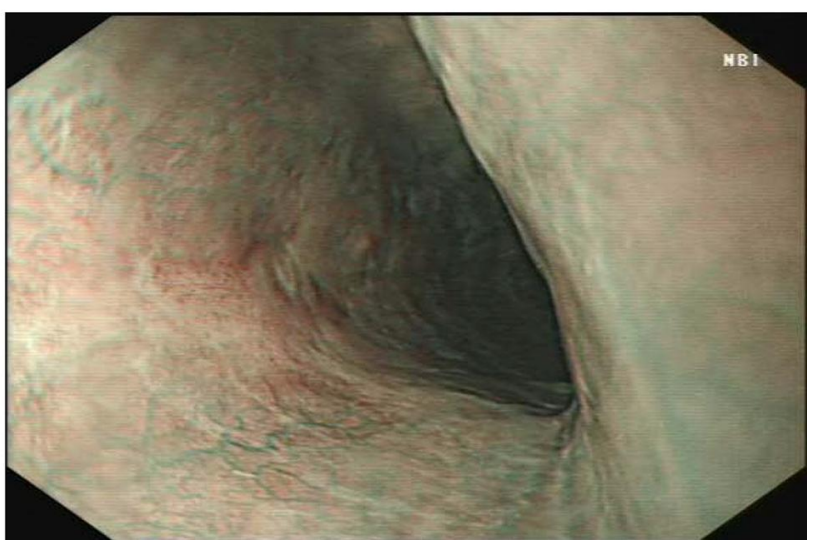

Figure 3 Gastroscopy during therapy. (A) SCCE relapse after six courses of IP chemotherapy. (B) Significant esophageal wall shrinkage after I month of anlotinib monotherapy.(C) Esophageal wall re-thickening following 3 months of anlotinib monotherapy. (D) Lugo's iodine staining of the esophageal wall 3 months after treatment with anlotinib in combination with toripalimab.

\section{Discussion}

In 2019, anlotinib was approved by the CSCO as a thirdline treatment option for esophageal squamous cell carcinoma. In 2020, anlotinib was also approved to treat SCLC. However, cases of SCCE treatment with anlotinib have not been reported yet. After two lines of treatment, the patient refused further chemotherapy treatment. Additionally, NGS testing did not indicate any typical mutations. However, NGS showed a high RB1 mutation rate accompanied by TP53 mutation, which is common in advanced SCCE,${ }^{16}$ and it indicates tumor growth due to chemotherapy resistance. ${ }^{17}$ Additionally, a high mutation of CTNNB1, ${ }^{18,19}$ which plays a critical role in tumor metastasis, might lead to innate primary resistance to ICIs, meaning immunotherapy may be unsuitable to use at that time. The high mutations in TP53 and TNFRSF8 ${ }^{20,21}$ indicated that anlotinib therapy may be beneficial.
According to both the cancer type and pathological type, anlotinib, as a novel multi-target tyrosine kinase inhibitor for tumor angiogenesis and proliferative signaling, was heavily recommended and chosen as the next line of therapy.

To the best of our knowledge, this is the first report about the successful use of anlotinib for advanced SCCE, which is characterized by the efficacy of CRand3 months of PFS. Interestingly, after anlotinib therapy, NGS showed changes that provided important information for guiding the next line of treatment. After anlotinib therapy, the KMT2A mutation disappeared and PTK2 amplified, which indicated an inhibition of metastasis and was interpreted as the patients benefiting from anlotinib therapy. ${ }^{22}$ As the mutation rate of CSF1R increased, the CTNNB1 mutation disappeared and MYC was amplified, ${ }^{23}$ which likely indicated a 
A

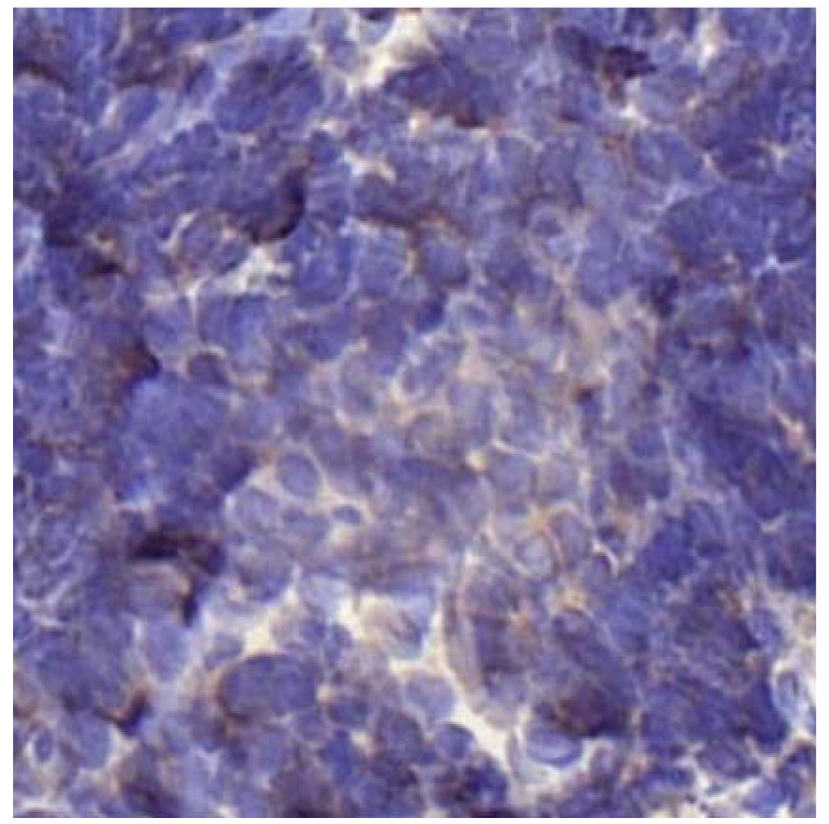

B

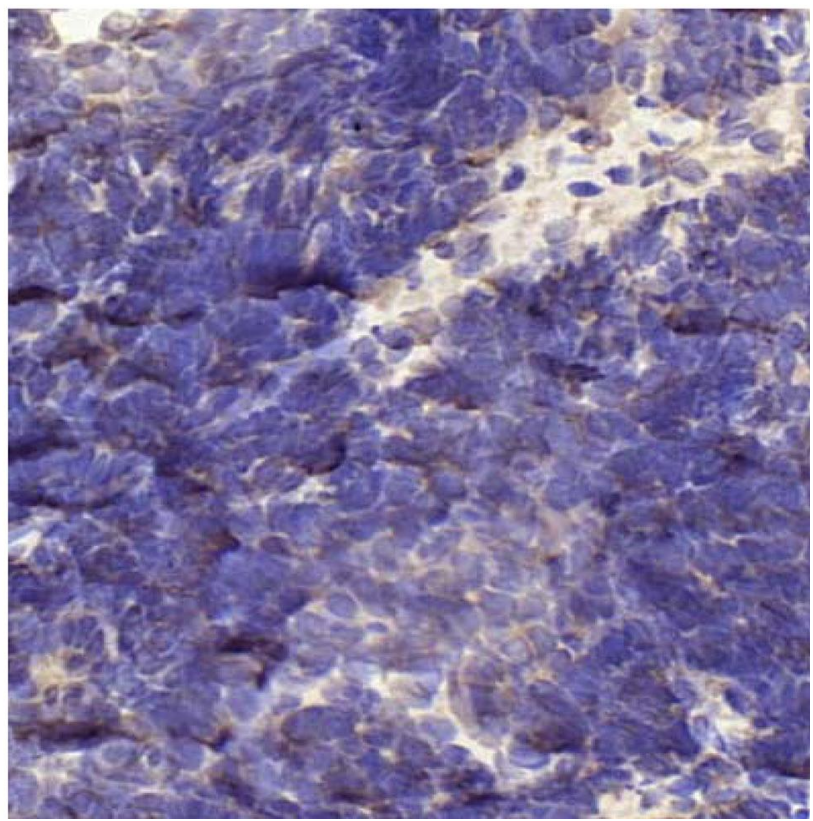

Figure 4 (A and B) IHC of PD-LI-positive expression in tumor biopsy specimens (brown). (A) Before anlotinib therapy, TPS < I\%; (B) after anlotinib therapy, TPS $\geq$ I\%.

resistance to anlotinib. A high TMB (57.1 mutations/ $\mathrm{Mb}$ ) indicated a potential benefit from ICI. According to the results mentioned above, the patient had a PD-L1 expression of more than $1 \%$, so anlotinib combined with toripalimab was selected as the next line of treatment.

According to the results of the GO and KEGG enrichment analyses of DEGs before and after anlotinib therapy, we deemed the FAK signal may be the main target for providing this patient with individualized treatment. FAK is known to be over expressed and activated in SCLC. ${ }^{24,25}$ FAK is critical in cancer development and progression because of its influence on multiple biological processes, such as crosstalk between the cell and its microenvironment, cell growth, survival, adhesion, spreading, migration, invasion, angiogenesis, DNA damage repair, radio resistance, and regulation of cancer stem cells. It has been reported that the over expression of FAK in vascular endothelial cells promotes angiogenesis, and the vascular endothelial growth factor inhibitor usually inhibits FAK. Since FAK inhibition could decrease vascular permeability and tumor vasculature, as well as prevent tumor growth, metastasis, and immunosuppressive tumor infiltration by cells, especially $\mathrm{T}$ regulatory cells, a clinical trial evaluating the association of FAK (VS6063) and PD-1 (pembrolizumab)inhibitors is ongoing. The results of the clinical trial may provide more evidence for the combination of antiangiogenic therapy combined with PD-1 in malignant tumors.

At the time of publication, the patient has remained in $\mathrm{CR}$, although PFS has not yet been reached after a 13 month follow-up (PFS > 13 months).

The present case study revealed an outcome of CR that is characterized by imaging examinations after 3 months of treatment, as well as good tolerance without significant adverse reactions. Our clinical data suggest that the combined treatment of anlotinib and toripalimab is a viable option for advanced SCCE that is resistant to chemotherapy. To the best of our knowledge, this is the first report of the successful use of anlotinib in combination with toripalimab for the treatment of advanced SCCE. To verify the efficacy and safety of this combination therapy, additional clinical trials with a larger sample size are needed. Additionally, the mechanism of the immunomodulatory effects of anti-angiogenic drugs should be studied further. 


\section{A}

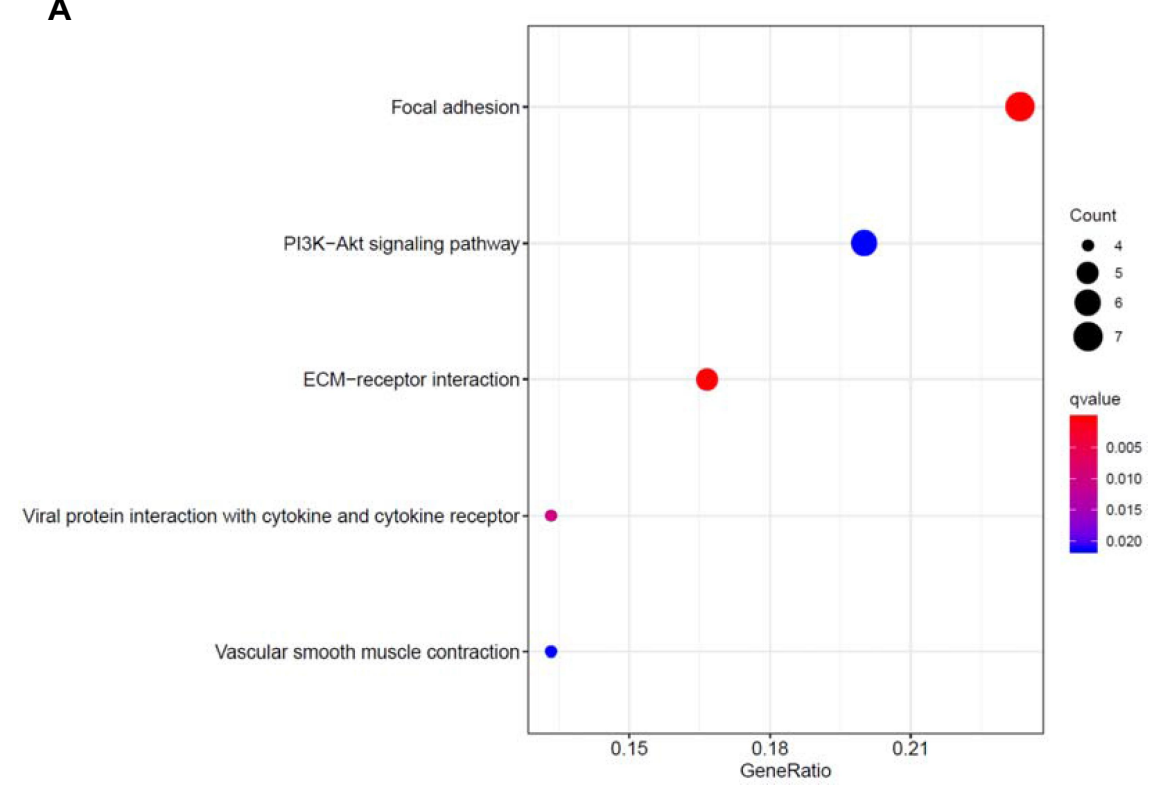

B

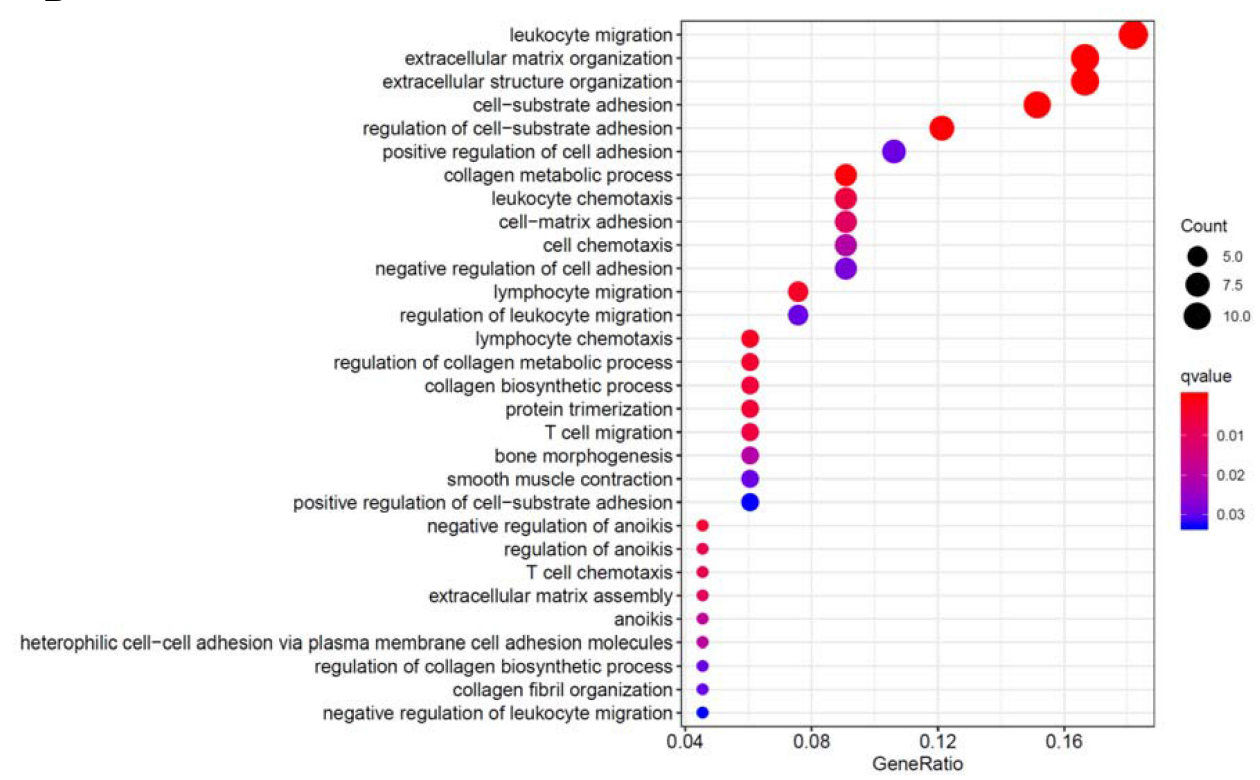

Figure 5 (A) Enrichment of the molecular function. (B) Enrichment of the cellular component (blue represents low aggregation; red represents high aggregation, and the larger the ball is, the more mutation genes it contains).

\section{Statement of Significance}

Until now, studies of small-cell carcinoma of the esophagus (SCCE) are lacking, and an optimal, standard treatment for limited-stage SCCE has not been established. In clinical practice, conventional radiotherapy and chemotherapy are generally ineffective for advanced SCCE, so a new treatment is urgently needed. Herein, we report the first successful case of an advanced-stage SCCE patient treated with the combination of anlotinib with toripalimab according to next- generation sequencing, which was proposed as a potential treatment for advanced SCCE.

\section{Ethics Statement}

The authors are accountable for all aspects of the work in ensuring that questions related to the accuracy or integrity of any part of the work are appropriately investigated and resolved. The study was approved by the institutional ethics committee in the affiliated 
hospital of Qingdao University (No. QYFYKYLL 471311920).

\section{Consent Statement}

The patient mentioned in this paper has signed written informed consent and confirmed her agreement to the publication of all case details and accompanying images.

\section{Acknowledgment}

The authors thank the patient and the investigators who made this study possible.

\section{Author Contributions}

Man Jiang, acquisition of data; analysis and interpretation of data; drafting of the manuscript; Xiaochun Zhang, study concept and design; acquisition of data. All authors made a significant contribution to the work reported, whether that is in the conception, study design, execution, acquisition of data, analysis and interpretation, or in all these areas; took part in drafting, revising or critically reviewing the article; gave final approval of the version to be published; have agreed on the journal to which the article has been submitted; and agree to be accountable for all aspects of the work.

\section{Funding}

This work was supported by the Taishan Scholar Foundation (grant tshw201502061 to X.Z.), Qingdao People's Livelihood Science and Technology Program (grant 16-6-2-3-nsh to X.Z and 18-2-2-74-jch to M J.), Chinese Postdoctoral Science Foundation (2017M6122218 to $\mathrm{M} \mathrm{J}$ ), Major innovation projects in Shandong Province (grant 2018cxgc1204 to X.Z).

\section{Disclosure}

The authors declare no potential conflicts of interest.

\section{References}

1. Bray F, Ferlay J, Soerjomataram I, Siegel RL, Torre LA, Jemal A. Global cancer statistics 2018:GLOBOCAN estimates of incidence and mortality worldwide for 36cancers in 185 countries. CA Cancer J Clin. 2018;68:394-424. doi:10.3322/caac.21492

2. Medgyesy CD, Wolff RA, Putnam JB, Alani JA. Small cell carcinoma of the esophagus: the University of Texas M.D. Anderson Cancer Center experience and literature review. Cancer. 2000;88:262-267. doi:10.1002/(SICI)1097-0142(20000115)88:2<262::AID-CNCR3>3.0. $\mathrm{CO} ; 2-\mathrm{K}$

3. Bennouna J, Bardet E, Deguiral P, Douillard JY. Small cell carcinoma of the esophagus: analysis of 10 cases and review of the published data. Am J Clin Oncol. 2000;23:455-459. doi:10.1097/00000421200010000-00005
4. Chow V, Law S, Lam KY, Luk JM, Wong J. Telomerase activity in small cell esophageal carcinoma. Dis Esophagus. 2001;14:139-142. doi:10.1046/j.1442-2050.2001.00172.x

5. Mitani M, Kuwabara Y, Shinoda N, Sato A, Fujii Y. Longterm survivors after the resection of limited esophageal small cell carcinoma. Dis Esophagus. 2000;13:259-261. doi:10.1046/j.1442-2050.20 00.00124.X

6. Zhu Y, Qiu B, Liu H, Xiao W, Hu Y, Liu M. Primary small cell carcinoma of the esophagus: review of 64 cases from a single institution. Dis Esophagus. 2014;27:152-158. doi:10.1111/dote.12069

7. Pantvaidya GH, Pramesh CS, Deshpande MS, Jambhekar NA, Sharma S, Deshpande RK. Small cell carcinoma of the esophagus: the Tata Memorial Hospital experience. Ann Thorac Surg. 2002;74:1924-1927. doi:10.1016/S0003-4975(02)04061-4

8. Xiao Q, Xiao H, Ouyang S, Tang J, Zhang B, Wang H. Primary small cell carcinoma of the esophagus: comparison between a Chinese cohort and surveillance, epidemiology, and end results (SEER) data. Cancer Med. 2019;8(3):1074-1085. doi:10.1002/cam4.2001

9. Wong AT, Shao M, Rineer J, Osborn V, Schwartz D, Schreiber D. Treatment and survival outcomes of small cell carcinoma of the esophagus: an analysis of the National Cancer Data Base. Dis Esophagus. 2017;30(2):1-5. doi:10.1111/dote.12487

10. Chen BQ, Yang H, Ma HL, et al. Radiotherapy for small cell carcinoma of the esophagus: outcomes and prognostic factors from a retrospective study. Radiat Oncol. 2019;14:210-217. doi:10.1186/ s13014-019-1415-9

11. Yu C, Liu X, Yang J, et al. Combination of immunotherapy with targeted therapy: theory and practice in metastatic melanoma. Front Immunol. 2019;7(10):990. doi:10.3389/fimmu.2019.00990

12. Makker V, Rasco D, Vogelzang NJ, et al. Lenvatinib plus pembrolizumab in patients with advanced endometrial cancer: an interim analysis of a multicentre, open-label, single-arm, Phase 2 trial. Lancet Oncol. 2019;20(5):711-718. doi:10.1016/S1470-2045(19)30020-8

13. Prelaj A, Tay R, Ferrara R, Chaput N, Besse B, Califano R. Predictive biomarkers of response for immune checkpoint inhibitors in non-small-cell lung cancer. Eur J Cancer. 2019;106:144-159. doi:10.1016/j.ejca.2018.11.002

14. Shen G, Zheng F, Ren DF, et al. Anlotinib: a novel multi-targeting tyrosine kinase inhibitor in clinical development. $J$ Hematol Oncol. 2018;11:120-127. doi:10.1186/s13045-018-0664-7

15. Lin B, Song X, Yang D, Bai D, Yao Y, Lu N. Anlotinib inhibits angiogenesis via suppressing the activation of VEGFR2, PDGFRb and FGFR1. Gene. 2018;654:77-86. doi:10.1016/j.gene.2018.02.026

16. Knudsen ES, Pruitt SC, Hershberger PA, Witkiewicz AK, Goodrich DW. Cell cycle and beyond: exploiting new RB1 controlled mechanisms for cancer therapy. Trends Cancer. 2019;5(5):308-324. doi:10. 1016/j.trecan.2019.03.005

17. Derks JL, Leblay N, Thunnissen E, et al. Molecular subtypes of pulmonary large-cell neuroendocrine carcinoma predict chemotherapy treatment outcome. Clin Cancer Res. 2017;24(1):33-42. doi:10. 1158/1078-0432.CCR-17-1921

18. Attar N, Kurdistani SK. Exploitation of EP300 and CREBBP lysine acetyltransferases by cancer. CSH Perspect Med. 2017;7: a026534.

19. Wen J, Min XJ, Shen M, et al. ACLY facilitates colon cancer cell metastasis by CTNNB1. J Exp Clin Cancer Res. 2019;38:401. doi:10.1186/s13046-019-1391-9

20. Dong ZY, Zhong WZ, Zhang XC, et al. Potential predictive value of TP53 and KRAS mutation status for response to PD-1 blockade immunotherapy in lung adenocarcinoma. Clin Cancer Res. 2017;23(12):3012-3024. doi:10.1158/1078-0432.CC R-16-2554

21. Dabir S, Kresak A, Yang M, Fu PF, Wildey G, Dowlati A. CD30 is a potential therapeutic target in malignant mesothelioma. Mol Cancer Ther. 2015;14(3):740-746. doi:10.1158/1535-7163.MCT14-0972 
22. Ma GX, Liu HT, Hua QH, et al. KCNMA1 cooperating with PTK2 is a novel tumor suppressor in gastric cancer and is associated with disease outcome. Mol Cancer. 2017;16:46-56. doi:10.1186/s12943017-0613-z

23. Haikala HM, Anttila JM, Marques E, et al. Pharmacological reactivation of MYC-dependent apoptosis induces susceptibility to anti-PD-1 immunotherapy. Nat Commun. 2019;10:620-637. doi:10.1038/s414 67-019-08541-2
24. Ocak S, Yamashita H, Udyavar AR, et al. DNA copy number aberrations in small-cell lung cancer reveal activation of the focal adhesion pathway. Oncogene. 2010;29:6331-6342. doi:10.1038/onc.2010.362

25. Park JH, Lee BL, Yoon J, et al. Focal adhesion kinase (FAK) gene amplification and its clinical implications in gastric cancer. Hum Pathol. 2010;41:1664-1673. doi:10.1016/j.humpath.2010.06.004

\section{Publish your work in this journal}

OncoTargets and Therapy is an international, peer-reviewed, open access journal focusing on the pathological basis of all cancers, potential targets for therapy and treatment protocols employed to improve the management of cancer patients. The journal also focuses on the impact of management programs and new therapeutic

Submit your manuscript here: https://www.dovepress.com/oncotargets-and-therapy-journal agents and protocols on patient perspectives such as quality of life, adherence and satisfaction. The manuscript management system is completely online and includes a very quick and fair peer-review system, which is all easy to use. Visit http://www.dovepress.com/ testimonials.php to read real quotes from published authors. 